\title{
Surgeon volume and 30 day mortality for brain tumours in England
}

\begin{abstract}
Matt Williams ${ }^{\star}, 1,2$, Peter Treasure ${ }^{3,4}$, David Greenberg ${ }^{3}$, Andrew Brodbelt ${ }^{5}$ and Peter Collins ${ }^{6}$ on behalf of the (UK) National Cancer Information Network Brain Tumour Group

${ }^{1}$ Department of Radiotherapy, Imperial College Healthcare NHS Trust, Charing Cross Hospital, Fulham Palace Road, London W6 8RF, UK; ${ }^{2}$ Computational Oncology Laboratory, Institute of Global Health Innovation, Faculty Building, South Kensington Campus, Imperial College, London SW7 2AZ, UK; ${ }^{3}$ National Cancer Registration and Analysis Service, Public Health England, Victoria House, Capital Park, Fulbourn, Cambridge CB21 5XA, UK; ${ }^{4}$ Department of Public Health and Primary Care, University of Cambridge, Institute of Public Health, Cambridge CB2 2SR, UK; ${ }^{5}$ Department of Neurosurgery, The Walton Centre NHS Foundation Trust, Lower Lane, Liverpool L9 7LJ, UK and ' Department of Pathology, University of Cambridge, Cambridge University Hospitals NHS Foundation Trust, Hills Road, Cambridge CB2 00Q, UK
\end{abstract}

Background: There is evidence that surgeons who perform more operations have better outcomes. However, in patients with brain tumours, all of the evidence comes from the USA.

Methods: We examined all English patients with an intracranial neoplasm who had an intracranial resection in 2008-2010. We included surgeons who performed at least six operations over 3 years, and at least one operation in the first and last 6 months of the period.

Results: The analysis data set comprised 9194 operations, 163 consultant neurosurgeons and 30 centres. Individual surgeon volumes varied widely $(7-272$; median $=46) .72 \%$ of operations were on the brain, and 30 day mortality was $3 \%$. A doubling of surgeon load was associated with a $20 \%$ relative reduction in mortality. Thirty day mortality varied between centres $(0 \cdot 95-8 \cdot 62 \%)$ but was not related to centre workload.

Conclusions: Individual surgeon volumes correlated with patient 30 day mortality. Centres and surgeons in England are busier than surgeons and centres in the USA. There is no relationship between centre volume and 30 day mortality in England. Services in the UK appear to be adequately arranged at a centre level, but would benefit from further surgeon sub-specialisation.

Primary brain tumours are rare, but are the leading cause of cancer death in those under 40 years old (Burnet et al, 2005). Surgical resection is a key modality in their management, and may either be curative or is a significant prognostic factor for malignant tumours (Bleehen and Stenning, 1991; Stupp et al, 2009; Stummer et al, 2011). Peri-operative death is a measure of clinical performance for surgeons, and is an easily understood measure of risk for patients. UK guidelines suggest that tumour surgery should be performed by a surgeon who spends at least $50 \%$ of their time devoted to neurooncology (NICE, 2006), but this is not always implemented.
There is evidence for a relationship between the number of operations a surgeon performs (surgeon volumes), the number of operations performed at a centre (centre volumes) or a combination of the two (patient volumes) and patient outcomes across a range of surgical procedures and specialities (Chowdhury et al, 2007). However, much of this evidence is based on limited areas of medical practice, with different definitions of volume, outcomes, procedures (oesophagectomy, colectomy, pneumonectomy and cardiac surgery) and diseases (all cause and cancer only) across the literature (Davoli et al, 2005; Bilimoria et al, 2008). Neurosurgical

*Correspondence: Dr M Williams; E-mail: matthew.williams2@imperial.nhs.uk

Received 3 April 2016; revised 9 August 2016; accepted 16 August 2016; published online 20 October 2016 
services in England are concentrated in relatively few tertiary referral centres which typically serve a population of $\sim 2$ million people, and so surgeon and centre volumes are relatively high compared with international comparisons, although variation still exists between centres and surgeons.

\section{METHODS}

We included all patients resident in England diagnosed with an intracranial neoplasm (primary brain, meningeal, pituitary, and metastasis) who underwent an intracranial debulking operation between 1 January 2008 and 31 December 2010 (list of procedures: Supplementary Appendix 1). We did not include patients having a cerebral biopsy, spinal operation, transsphenoidal operation or craniotomy alone (with no resection). In patients who had undergone more than one operation, only the latest operation was examined. To only include active neurooncology surgeons, we excluded surgeons who had performed $<6$ operations over this period (an average of one operation per 6 months), or who had not performed at least one operation in the first 6 months of 2008 and the last 6 months of 2010 . Speciality status of included surgeons was confirmed by checking on the national register of specialists. Deprivation quintile was calculated based on patient household index of multiple deprivation, calculated in 2010 (IMD 2010). Vital status of patients was checked using the NHS Personal Demographics Service.

We assessed individual surgeon volume, centre volume, site of operation, patient age, sex and deprivation score as predictors of 30 day mortality. Mixed logistic regression models were constructed using these predictors as fixed effects, and surgeon and centre as random effects. The effect of centre volume was assessed using the number of operations performed at that centre, minus those conducted by the surgeon in question. For each factor a relative risk (RR) was calculated in relation to a baseline category, and we assessed statistical significance using likelihood tests. For centre and surgeon volumes, the RR of 30-day mortality was calculated for a doubling of workload. Univariate mixed logistic regression was conducted to assess the relationship between these variables and 30 day mortality, and we constructed a multivariate model based on these factors. Potential clustering effects of surgeon and centre were adjusted for by including surgeon and centre as random effects in all models. To assess the impact of including factors in a multivariate model, both the impact of adding factors and removing factors were examined.

To model the potential benefit of moving patients from a lower to higher volume setting, we modelled the impact of moving the patients who were operated on by surgeons operating less than once per month to those operating once per week, but assumed that the risk for these surgeons remained the same.

Given that this study was a retrospective study of routinely treated patients, it did not require specific ethical approval. However, this project was registered as part of the National Cancer Information Network's (NCIN) Brain Tumour Group annual work plan.

\section{RESULTS}

We identified 10888 patients over a 3 year period, of whom we included 9194 patients operated on by 163 eligible consultant surgeons in 30 centres. Individual surgical volumes ranged between 7 and 272 operations over 3 years (median $=46$; IQR: 29-70). $71.5 \%$ of operations were on the brain, rather than the meninges (6574 vs 2620). The majority of patients had either primary brain or meningeal tumours, and the commonest 5 -year age group was 60-65 years. Patient, tumour, surgeon and centre characteristics are summarised in Supplementary Tables 1 and 2.

The crude 30-day mortality for the whole cohort was 3.01\% and for the analysis data set it was $3.02 \%$. The crude 30 -day mortality was higher in for those surgeons with the least activity $(4.60 \%$ in the lowest quartile $(7-<29$ operations) vs $2.48 \%$ in those $\geqslant 70$ operations; Table 1).

On univariate analysis, individual surgeon, site of operation, patient age and patient deprivation quintile were predictors of 30 day mortality, whereas patient sex and centre volume were not (Table 1). The multivariate model included individual surgeon volume, site of operation and patient's age, sex and deprivation score. Thirty-day mortality was higher in older patients, in those who had an operation on the brain rather than the meninges, and if they came from a more deprived area (Table 1).

The number of operations per centre varied from 29 to 667 (median 273). There was substantial variation in 30-day mortality

Table 1. The effect of demographic, clinical and surgeon factors on 30-day mortality

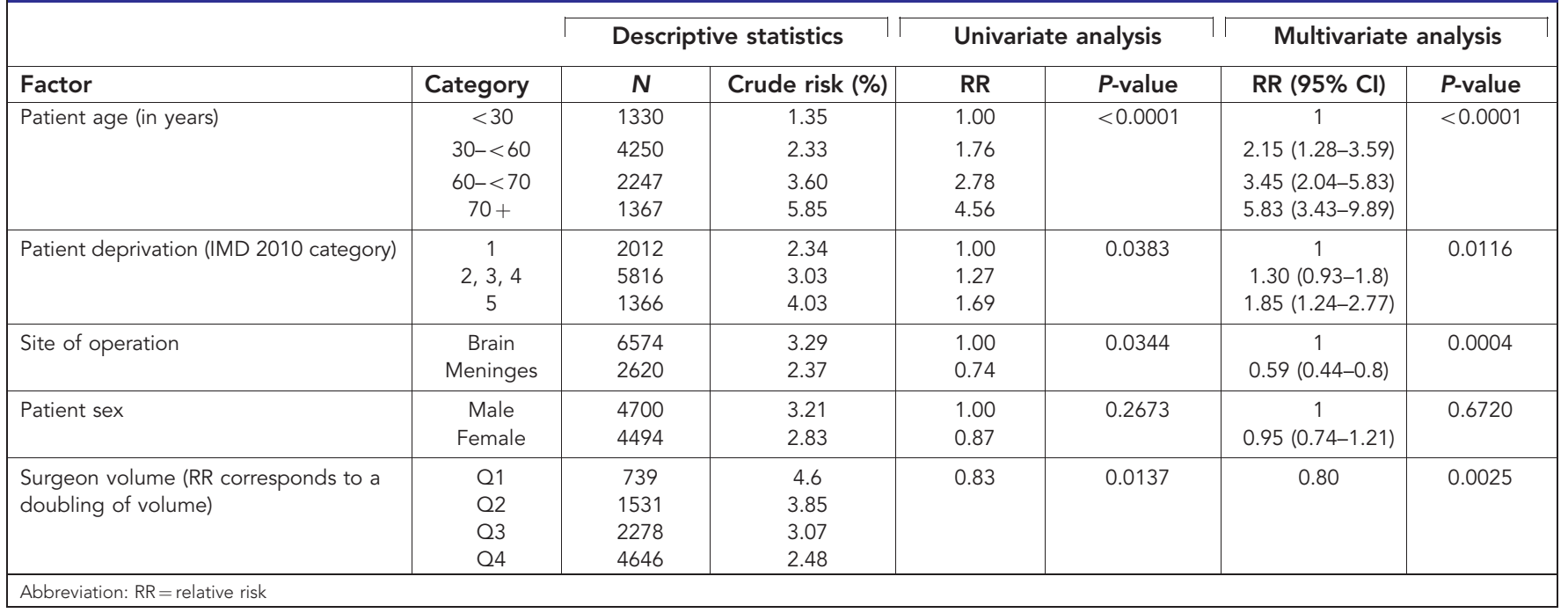


between centres-in the $80 \%$ of centres with more than 100 cases, the crude mortality varied between $0.95 \%$ and $8.62 \%$, but this did not correlate to centre volumes (Spearman's $r=-0.11$; $P=0.57$ ), and individual surgeon volume was not correlated with centre volumes (Spearman's $r=0.08 ; P=0.30$ ).

To estimate the effect of case-mix we used a model that included age, tumour type, surgery location and deprivation. Predicted mortality, based on these, was lower for those patients operated on by less-busy surgeons ( $<1$ per month) than those operated on by busier surgeons ( $\geqslant 1$ per week; $2.97 \%$ vs 3.13\%; Supplementary Table 3).

An unadjusted model (surgeon volume and random effects) showed that a doubling of volume was associated with a $17 \%$ reduction in risk $(0.95 \mathrm{CI}=(4 \%, 28 \%)$, likelihood ratio $P$-value $=0.014)$. In the multivariate adjusted model a doubling of volume was associated with a $20 \%$ reduction of risk $(0.95 \mathrm{CI}=(8 \%, 31 \%)$, likelihood ratio $P$-value $=0.0025$ ).

\section{DISCUSSION}

In patients undergoing surgical resection of a brain tumour in England the average 30-day mortality was 3\%. This was associated with surgeon volume, patient age and patient deprivation, and is a similar rate to other studies. On multivariate analysis there was a $20 \%$ relative reduction in risk for each doubling of workload, even though busier surgeons appeared to operate on patients with a slightly higher risk of death. There was no impact of centre volumes on 30 day mortality, although there was significant variation between centres. However, both centres and surgeons in England were considerably busier than in previous studies (Supplementary Table 3), which may explain the limited effect of centre volume.

The current study has considered only individual surgeon and centre volumes and a few clinical variables. Although we considered aggregate surgeon activity over a 3 year period rather than a rolling 365 day average, activity within this period is unlikely to substantially fluctuate. Data on comorbidities and tumour site were not available, and neither were data on route of admission (emergency vs routine). Previous work has suggested that adding additional clinical information tends to reduce the magnitude of the effect of surgeon volumes (Panageas et al, 2003). In addition, in patients with high-grade tumours, the risk of peri-operative death is small compared with the risk of death over the 5 years following diagnosis.

All of the published studies on the relationship between patient volumes and outcomes in neuro-oncology are based on data from the USA (Supplementary Table 4) (Barker et al, 2003, 2005; Cowan et al, 2003; Long et al, 2003; Trinh et al, 2015). These studies did not adjust for clustering, and are notable for the small surgeon and centre volumes-the busiest $10 \%$ of surgeons and centres in USbased studies would fall near the median levels in the UK (Trinh et al, 2015), which may explain why their study found evidence of both centre and individual surgeon effects. In England \& Wales, the National Institute for Health and Care Excellence (NICE) recommended that surgeons spend 'at least $50 \%$ of (their time) in neuro-oncological surgery', and the national neurosurgical audit programme has published consultant outcomes since Dec 2014. No surgeon was reported as being outside control levels for mortality at the initial publication, but there was no analysis of the factors predicting outcomes, and no analysis of individual caseload to assess whether the $50 \%$ was met. It is unclear whether the $50 \%$ threshold is of value in neuro-oncological neurosurgery, and our current work does not address this at present. Given that the mortality rate is lower than many other procedures for which there is a volume effect, we suspect that many of the mechanisms by which surgeon volume influences 30-day mortality lie outside the operating theatre, and include patient selection and post-operative care.

Our final model predicts 50 deaths over three years amongst patients of surgeons who operate less than once per month. If these operations had been performed by a surgeon operating once per week the corresponding predicted number of deaths is 28, a reduction of $44 \%$, although the overall risk is low. It appears that work is already appropriately concentrated at a centre level, but should be concentrated in fewer surgeons in the same centres. This move towards sub-specialisation should be relatively easy to achieve, with minimal additional resource, although there needs to be additional work to understand the variation in mortality between centres. Taken with previous evidence, the implications of this work for other countries, where the workload is more widely spread, are more disruptive, and provide evidence for much more wide-ranging reorganisation of services.

\section{ACKNOWLEDGEMENTS}

A Brodbelt and P Collins received no funding for this work. $\mathrm{D}$ Greenberg and $\mathrm{P}$ Treasure receive funding from PublicHealth England for the data work used in this publication. M Williams was supported by the Brain Tumour Research Campaign.

\section{CONFLICT OF INTEREST}

The authors declare no conflict of interest.

\section{REFERENCES}

Barker FG, Curry WT, Carter BS (2005) Surgery for primary supratentorial brain tumors in the United States, 1988-2000: the effect of provider caseload and centralization of care. Neuro Oncol 7: 49-63.

Barker FG, Klibanski A, Swearingen B (2003) Transsphenoidal surgery for pituitary tumors in the United States, 1996-2000: mortality, morbidity, and the effects of hospital and surgeon volume. J Clin Endocrinol Metab 88: 4709-4719.

Bilimoria KY, Bentrem DJ, Feinglass JM, Stewart AK, Winchester DP, Talamonti MS, Ko CY (2008) Directing surgical quality improvement initiatives: comparison of perioperative mortality and long-term survival for cancer surgery. J Clin Oncol 26: 4626-4633.

Bleehen NM, Stenning SP (1991) A Medical Research Council trial of two radiotherapy doses in the treatment of grades 3 and 4 astrocytoma. The Medical Research Council Brain Tumour Working Party. Br J Cancer 64: 769-774.

Burnet NG, Jefferies SJ, Benson RJ, Hunt DP, Treasure FP (2005) Years of life lost (YLL) from cancer is an important measure of population burden-and should be considered when allocating research funds. Br J Cancer 92: 241-245.

Chowdhury MM, Dagash H, Pierro A (2007) A systematic review of the impact of volume of surgery and specialization on patient outcome. Br J Surg 94: 145-161.

Cowan JA, Dimick JB, Leveque J-C, Thompson BG, Upchurch GR, Hoff JT (2003) The impact of provider volume on mortality after intracranial tumor resection. Neurosurgery 52: 48-53discussion $53-54$.

Davoli M, Amato L, Minozzi S, Bargagli AM, Vecchi S, Perucci CA (2005) [Volume and health outcomes: an overview of systematic reviews]. Epidemiol Prev 29: 3-63.

Long DM, Gordon T, Bowman H, Etzel A, Burleyson G, Betchen S, Garonzik IM, Brem H (2003) Outcome and cost of craniotomy performed to treat tumors in regional academic referral centers. Neurosurgery 52: 1056-1063; discussion 1063-1065.

NICE (2006) Improving outcomes for people with brain and other central nervous system tumours (NICE). Available at: https://www.nice.org.uk/ guidance/csg10 (accessed 2 October 2016). 
Panageas KS, Schrag D, Riedel E, Bach PB, Begg CB (2003) The effect of clustering of outcomes on the association of procedure volume and surgical outcomes. Ann Intern Med 139: 658-665.

Stummer W, Van Den Bent MJ, Westphal M (2011) Cytoreductive surgery of glioblastoma as the key to successful adjuvant therapies: new arguments in an old discussion. Acta Neurochir (Wien) 153: 1211-1218.

Stupp R, Hegi ME, Mason WP, van den Bent MJ, Taphoorn MJB, Janzer RC, Ludwin SK, Allgeier A, Fisher B, Belanger K, Hau P, Brandes AA, Gijtenbeek J, Marosi C, Vecht CJ, Mokhtari K, Wesseling P, Villa S, Eisenhauer E, Gorlia T, Weller M, Lacombe D, Cairncross JG, Mirimanoff R-O (2009) Effects of radiotherapy with concomitant and adjuvant temozolomide versus radiotherapy alone on survival in glioblastoma in a randomised phase III study: 5-year analysis of the EORTC-NCIC trial. Lancet Oncol 10: 459-466.

Trinh VT, Davies JM, Berger MS (2015) Surgery for primary supratentorial brain tumors in the United States, 2000-2009: effect of provider and hospital caseload on complication rates. J Neurosurg 122: 280-296.

cc)(i)(2) This work is licensed under the Creative Commons (c) Attribution-Non-Commercial-Share Alike 4.0 International License. To view a copy of this license, visit http:// creativecommons.org/licenses/by-nc-sa/4.0/

Supplementary Information accompanies this paper on British Journal of Cancer website (http://www.nature.com/bjc) 